\title{
QoS Provisioning in a Service Oriented Architecture Targeting the Smart Grid World
}

\begin{abstract}
In today's liberalized market of energy, dynamic selections of services and by extension of service providers are crucial. It is equally important for Service Providers to spot the one QoS Module that offers the best QoS level in a given cost. Attributes like type of service, response time, throughput, availability and cost, form the basic set of attributes that should be taken into consideration when building a concrete/stable Grid network. In the proposed QoS architecture, Prosumers request services based on the aforementioned set of attributes. The Prosumer requests the service through a QoS Module. Afterwards, it is the QoS Module's responsibility to seek the Service Provider that best serves the needs of the client.
\end{abstract}

Keywords: QoS; Service Oriented Architecture; Smart Grid.

\section{Introduction}

Nowadays, in the market of energy playground is more crucial than ever to seamlessly provide the end users with the requested services, without putting in jeopardy the grid's stability. In this constantly growing and demanding market of energy environment, arises the need for providing an efficient Quality of Service (QoS) mechanism to adequately meet the constraints that are imposed by the consumers of energy, without on the same time neglecting the importance of maintaining the balance of energy flow on the network in an as stable as possible level.

In order to properly meet this target, an in advance way of placing, scheduling, and assigning the requests for energy consumption (or even for energy production) should be considered. A system based on attributes like: type of service to be served, response time, availability, cost and probably throughput should be explored, developed and adopted in order to easily pass from the classic obsoleted energy grid to this new more intelligently build Smart Grid.

In the proposed approach, we try to apply the Service Oriented Architecture Approach (SOA) to the Smart Grid world. The idea was emerged by noticing that in Smart Grid field the whole action is triggered by two main actors, namely the Consumer and the Provider of energy (the service). In our case the two main actors are the Prosumer/User and the Aggregator. This is the perspective from which the SOA is overlooking a system. So, we tried to get the best of what the SOA approach has to offer, in order different Providers to be able to independently create their services and seamlessly "feed" the Consumers. 
The rest of the paper is structured as follows. Section 2 gives an overview of what Smart Grid really is. In Section 3 the motivation for bringing QoS in SOAs and Smart Grids is described. Section 4 gives a detailed presentation of the proposed QoS approach, while Section 5 concludes the presented, and outlines future work.

\section{Smart Grid Overview}

The "smart grid" is an electrical power system that increases the use of communications and information technology in the generation, delivery and consumption of electrical energy with advance sensing technology and automatic control technology between the power and the infrastructure of the electricity grid. The Smart Grid consists of connections between suppliers, distributors and consumers. Control is a key factor in enabling technology for the deployment of renewable energy systems.

The use of smart grid system is an initiative to improve the development of clean energy and green technologies.

Efficient, reliable transmission and distribution of electricity is a fundamental requirement for providing societies and economies with essential energy resources. The general goals of Smart Grid are to ensure a transparent, sustainable and environmental friendly system operation that is cost and energy efficient, secure and safe. The objectives for deploying Smart Grids may differ from country to country, a common ground can be easily delimited in achieving the following:

- Robustness: The Smart Grid shall improve resilience to disruption to provide continuous and stable electricity flows, avoiding wide-area breakout accidents. It shall guarantee the normal and secure run of the electricity grid even under the instance of emergency issues, such as natural disasters, extreme weather and man-made breakage, and provides self-healing abilities;

- Secured operation: The Smart Grid shall enhance communication networks and information security of the electricity grid;

- Compatibility: The Smart Grid shall support the integration of renewable electricity such as solar and wind, has the capacity of distributed generation access and micro-grids, improve demand response functions, implement the effective twoway communication with consumers and satisfy various electricity demands of consumers;

- Economical energy usage: The Smart Grid shall have the capacity of more effective electricity markets and electricity trades, implement optimized configuration of resources, increase efficiency of the electricity grid, and reduce electricity grid wastage;

- Integrated system: The Smart Grid shall highly integrate and share information and data of an electricity grid, utilize the uniform platform and model to provide standardized and refined management;

- Optimization: The Smart Grid shall optimize assets, reduce costs and operate efficiently; 
- Green energy: The Smart Grid shall solve problems of energy security, energy saving, carbon dioxide emission and etc.

A smart grid should be able to integrate real-time information and optimal control methodology to provide location specific, real-time actions as well as solutions for smart consumption of power for the end-user consumers to conserve energy and cut their utility bills. The power system control can be facilitated by real-time data collection which is becoming readily available due to improvements in advanced information technology that make the smart grid a feedback control system.

- Observability: It enables the status of electricity grid to be observed accurately and timely by using advanced sensing and measuring technologies it enables the customers to know the amount of energy consume. This refers to smart meters and an accompanying communications network that allows two-way communication between the provider of electricity and the meter. This allows providers to have access to real-time information on the electricity consumption of each customer

- Controllability: It enables the effective control of the power system by observing the status of the electricity grid; because the amount of energy generated most equal the demand. A system is considered an optimum control system when the system parameters are adjusted so that the index reaches an extreme value, commonly a minimum value. With this the utility grid can effectively have control to over distribution in order to reduce overloading on the grid.

- Timely analysis and decision-making: It enables the improvement of intelligent decision-making process; it is observed that electricity is more expensive at peck period when the demand is high; with the smart grid as a feedback control system, the customers will be able to know when to use some specific appliances that consume more energy because of the interaction with the utility grid. This can also reduce demand during the high cost peak usage periods, communications and metering technologies inform smart devices in the home and business when energy demand is high and track how much electricity is used and when it is used. It also gives utility companies the ability to reduce consumption by communicating to devices directly in order to prevent system overloads.

- Self-adapting and self-healing: It prevents power disturbance and breakdown via self-diagnosis and fault location. Smart grid network is required to connect the magnitude of electric devices in distributed locations and exchange their status information and control instructions. A monitoring sensor system conditions at a control center can simulate various system wide corrective actions in less than half a second and send instructions to control computer throughout the system. When a failure is detected, circuit breakers are triggered to isolate the problem and prevent other lines from being damaged; the circuit breaker prevents the substation from damage when there is power surge.

\section{QoS in Service Oriented Architectures}

SOA is a way of developing software in the form of interoperable services. The promise that the service-oriented development brings to the IT world stems in providing a common programming interface, through which any application can be 
accessed [1]. A service can be defined as a discrete unit of functionality that is made available through a service contract [2]. The service contract specifies all interactions between the service consumer(s) and service provider(s) and includes: i) Service interface; ii) Interface documents; iii) Service policies; iv) Quality of service (QoS); and v) Performance.

One of the main differences between a service and other software constructs (such as components or objects) is that a service is explicitly managed. The QoS and performance are managed through a service level agreement (SLA). In addition, the entire service life cycle is managed - from design, to deployment, to enhancements, to maintenance.

SOAs can easily support QoS features and behavior by putting their characteristics in the WSDL description of a requested or provided service. Since SOAs message exchange is based on XML, we only need to flourish a bit the description in order to make it possible.

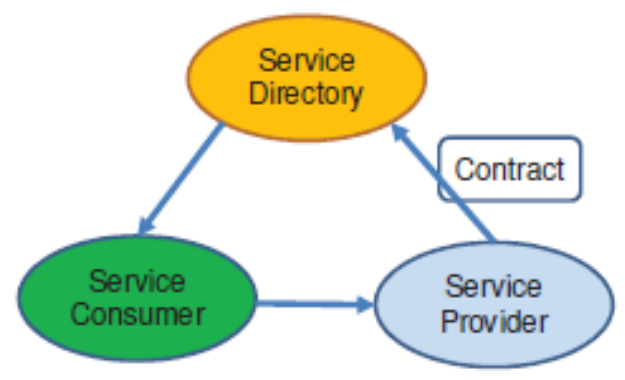

Fig. 1. Service-Oriented Architecture overview.

Normally the need for code and systems re-use is the driving force for adopting SOAs [3] instead of using highly specialized building blocks, focusing on a certain application. A service must hide its internal logic. A service should be loosely coupled, with no predefined connections.

\section{Proposed Architecture}

The QoS architecture presented in the paper is consisted of the following components: the Aggregator, the Aggregator Agent (AA), the Prosumer/User, the Flex-Offer Agent (FOA) [4], the QoS Agent, the Aggregator Registration, and databases: to store information regarding the Prosumers/Users, the Contracts (closed, served, etc.), and information regarding the available Aggregators and their characteristics. See Figure 2. 


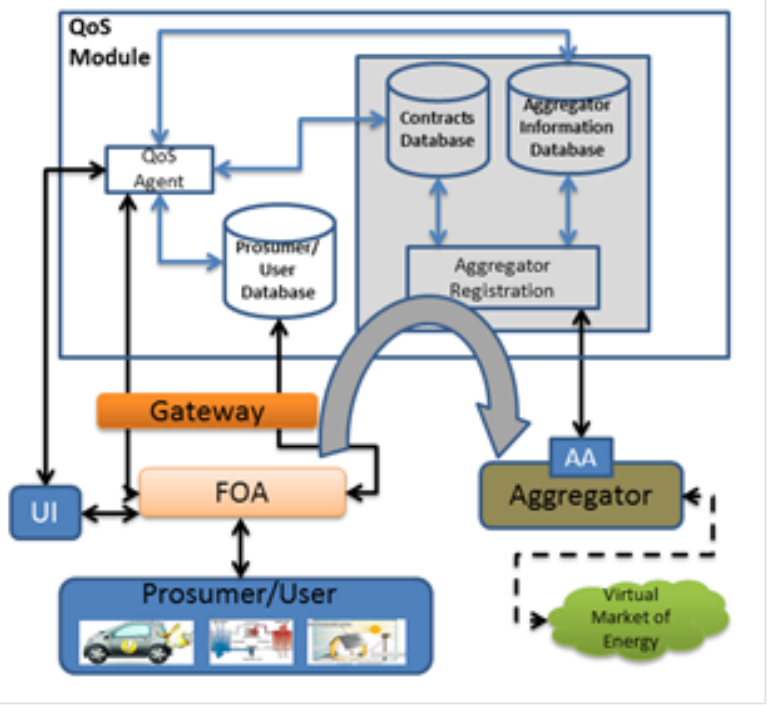

Fig. 2. Proposed QoS Architecture.

The Prosumers/Users send their micro flex-offers to the Aggregator, through the FOA and QoS Module. A micro flex-offer states the possibility of a Prosumer/User to consume a certain amount of energy and the time interval during which it has the flexibility to schedule that consumption. There is also the possibility the flex-offer to be generated by the Flex-Offer Agent or by a Flex-Offer Agent that resides on the Aggregator's side, but we will not consider these two options in the present work.

The Aggregators are capable of joining several micro flex-offers into larger macro flex-offers, which are then placed on the electricity market. The energy market will answer with bids to buy and sell energy at given times. Aggregators receive and respond to the bids which allocate energy consumption periods to the macro flexoffers. After, they disaggregate macro flex-offer responses and send an answer to the Prosumers/Users which specify the periods of time to consume the required energy amount from the grid at a lower cost. It is the QoS Module that has the responsibility to find the best matching between the Prosumer's request for a service and the Aggregator that best covers its needs, in terms of response time, availability, and cost.

\subsection{Aggregator}

The Aggregator is responsible for the handling of flex-offers from the FOA, joining (aggregating) several micro flex-offers into a larger macro flex-offer, placing the macro flex-offer on the Virtual Market of Energy, disaggregating scheduled macro flex-offers, sending scheduled micro flex-offers to FOAs, controlling the 
execution of a micro scheduled flex-offer, determine if the execution of the flex-offer by the Device had been done according to the scheduled flex-offer. Each Aggregator can be specialized on different types of devices, by running the most adequate algorithms for the aggregation and disaggregation of flex-offers.

\subsection{Aggregator Agent (AA)}

Aggregator has an agent that provides information to the QoS Module. The Aggregator provides information to the QoS Module that has to do with the number of the users he is able to serve, possible cost of the provided service, time to respond to the Prosumer's request. He can also provide information regarding the type of services he can provide. It is common in the energy market the existence of different type of Aggregators to cover the needs for home appliances (e.g., washing machines, heat pumps, etc.) and different ones to cover the needs of Electric Vehicles charging. This information is of great importance to the QoS Module in order to correctly and fast spot the most appropriate Aggregator to deliver the service to the Prosumer. The AA is indirectly connected to Flex-Offer Agent (FOA) via the QoS Module.

\subsection{Aggregator Registration}

Aggregator Registration allows Aggregators, through the Aggregator Agent (AA) to submit: their id, service descriptions, cost functions, availability, and number of Prosumers/Users they can serve, to the QoS Module.

\subsection{Prosumer/User}

A Prosumer (or User) owns Devices ${ }^{1}$ and has an agreement with an Aggregator regarding utilizing the devices power consumption or production flexibility. The Prosumer has to set up all relevant constraints/comfort requirements, which the flexoffer must fulfill. The Prosumer might be a household, factory, an office building, i.e. a legal entity that owns devices. A Prosumer uses a Flex-Offer Agent to generate flexoffers or he can configure those parameters through a user interface.

\subsection{Flex-Offer Agent}

Every Prosumer/User has an agent that provides information to the QoS Module. FOA is a software module, which acts as an intermediate between Devices and Aggregators, being able to be executed on a variety of hardware platforms and of

${ }^{1}$ Devices are the end equipment that consume or produce the energy belonging to a flex-offer, e.g., a refrigerator or a washing machine. Devices can have the capability of being remotely controlled or might not have any computer interfacing capabilities. Devices can be classified in two types: FOA-enabled Devices and Legacy Devices (that need a switch and a power meter in order to obtain the needed intelligence). 
easily being configured to use different protocols. Based on constraints set up by the Prosumer and on power consumption measurements taken from devices it uses a specific algorithm to automatically generate micro flex-offers. Other inputs like weather forecasts might also be used. The FOA can send the micro flex-offers to the Aggregator and receive the micro scheduled flex-offers from it. Another kind of information the Prosumer/User passes to the QoS is the type of service he needs (domestic appliances, heat pumps, or EVs). As in the case of the Aggregator Agent, this information is of great importance to the QoS Module in order to correctly and fast spot the most appropriate Aggregator to deliver the service to the Prosumer. The Flex-Offer Agent passes the request for a service to the QoS Module through the QoS Agent.

\subsection{QoS Agent (QA)}

QoS Agent (QA) is responsible to evaluate the Prosumer request, and identify an Aggregator that properly meets the client's needs. QoS Agent receives the request from the Flex-Offer Agent (FOA) and evaluates the Prosumer/User request against each available Aggregator in order to spot the one that best fits the Prosumer/User needs. A Prosumer's request will probably contain a service type, cost constraint and the preferred comfort level. By the time the mapping is succeeded the micro flex-offer is passed to the Aggregator to continue with the building of the macro flex-offers and the placement to the market of energy

\subsection{User Interface}

User Interface can take care of the interactions among the Prosumers/Users, the FOA, and QoS Agent through a web-based interface. It can be used to allow generation of flex-offers by a Prosumer/User or just to enforce attributes like a particular comfort level to the QoS Module.

\subsection{Gateway}

Gateway can be seen as a device that converts between the protocols used internally on a Home Area Network and the internet. It is possible to have the capability of executing the Flex-Offer Agent.

\subsection{Contracts and Aggregator Information Databases}

Contracts Information Database is a database to store SLAs, closed, scheduled, and served contracts. Aggregator Information database is a database for keeping information regarding the Aggregators, Aggregator's information like type of services, availability, response time and cost models. Also the id of the Aggregator is stored on the Aggregators Information database. The id of the Aggregator is important 
in order the Prosumer/User through the FOA, and the QoS Module to spot the correct one.

\subsection{Prosumer/User Database}

Prosumer/User Database is a database that holds information regarding the Prosumers/Users. Information like: power consumption, type of Prosumer/User (flexoffer enabled or legacy device), if he was served or not.

\subsection{QoS Module Interactions}

The available Aggregators register themselves to the QoS Module and particular to the Aggregators Information Database, providing information like type of provided services, response time and cost models. The Prosumer asks for a service, which in our case is a need for energy consumption. This type of information is named micro flex-offer. It is then responsibility of the QoS Module to perform all the needed steps in order to spot the Aggregator that best serves the needs of the Prosumer.

\subsection{QoS Delivery}

Figure 3 presents the interactions between the Prosumer, the QoS Module and the Aggregator

1. Aggregators register themselves (with their id), and their services (type of services, response time, cost models, and number of Prosumers/Users each can serve) to the QoS Module.

2. A Prosumer/User initiates the sequence of steps, by sending to the QoS Module a QoS request (pointing out the requested service type, amount of needed energy, cost constraints, time flexibility).

3. The QoS Module spots the Aggregator that best fits the needs of the Prosumer/User. The QoS Module creates a token that includes information like the id of the Aggregator, a session id, the service id, expiration date and time for the offer.

4. If the Prosumer accepts the offer, the QoS Module saves it in the Contract database. The Prosumer only needs the created token to request the service in the given time.

5. The Prosumer makes a service request to the Aggregator using the created token.

6. The Aggregator creates the macro flex-offer and places a bid to the Virtual Market of Energy. The market answers back with a schedule.

7. The Aggregator sends the Schedule to the Prosumer/User, through the FlexOffer Agent.

8. The Prosumer consumes the service and reports back to the Aggregator the power consumption. 


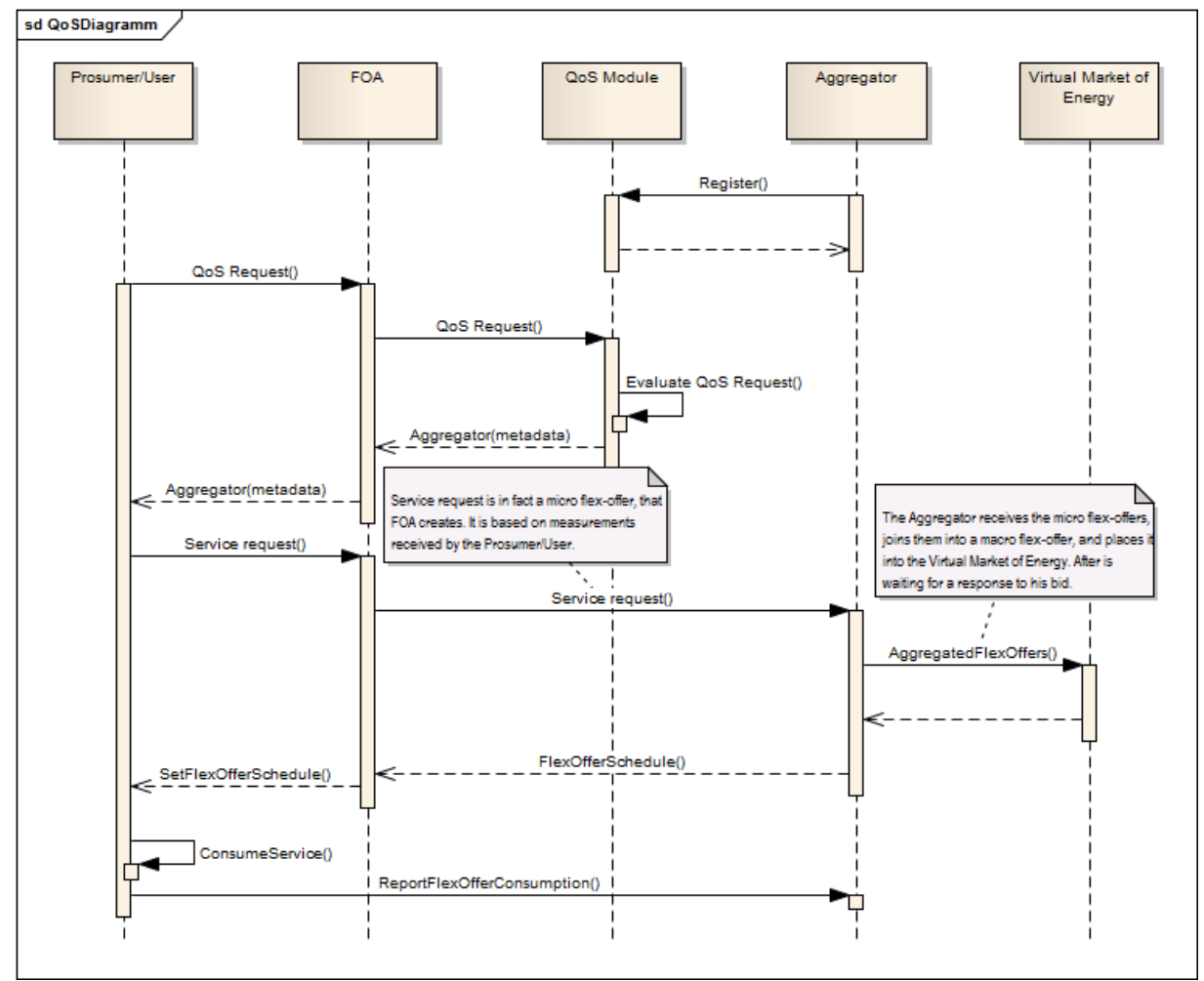

Fig. 3. QoS Delivery Sequence Diagram.

\section{Conclusions}

In this article we have presented our initial thoughts for a Quality of Service architecture targeting the Smart Grid world. All the involving parts were in detail described and documented. QoS attributes like: type of service to be served, response time, availability, and cost where taken into consideration while sketching the proposed architecture. Future work will include definition of algorithms to be used for the QoS provisioning and implementation of the proposed architecture. Another equally important step is handling the different ways that a flex-offer can be generated and come up with an as common as possible approach. In this paper we considered the flex-offer to be created by the Flex-Offer Agent that is actually connected to the Prosumer/User. The other two spotted formal cases are the generation of the flex-offer on the Aggregator, by using power measurement data available on the cloud, and the last but not least the flex-offer to be initiated by the User, through a User Interface provided by the Flex-Offer Agent. 


\section{References}

1. Newcomer, E., Lomow, G.: Understanding SOA with Web Services, ISBN-10: 0321180860, ISBN-13: 9780321180865, Publisher: Addison-Wesley Professional, (2005)

2. Rosen, M., Lublinsky, B., Smith, T.K., Balcer, J.M.: Applied SOA: Service-Oriented Artchitecture and Design Strategies; John Wiley \& Sons; Pub. Date: June 16, 2008, Print ISBN: 978-0-470-22365-9; Web ISBN: 0-470223-65-0 (2008)

3. Thomas, E.: SOA Design Patterns, Prentice Hall PTR, ISBN: 0136135161 (2009)

4. Ferreira, L., Siksnys, L., Pedersen, P., Stluka, P., Chrysoulas, C., Guilly, T., Albano, M., Skou, A., Teixeira, C., Pedersen, T.: Arrowhead compliant virtual market of energy, in Emerging Technology and Factory Automation (ETFA), 2014 IEEE, Sept 2014, pp. 1-8 (2014) 